\title{
Are there carbon stars in the Bulge?
}

\author{
Yuen Keong $\mathrm{Ng}$ \\ Padova Observatory, Vicolo dell'Osservatorio 5, I-35122 Padua, Italy
}

\begin{abstract}
The Bulge carbon stars have been a mystery since their discovery by Azzopardi et al. (1991), because they are about $2^{m} .5$ too faint to be regarded as genuine AGB stars, if located inside the metal-rich Bulge $\left(\mathrm{m}-\mathrm{M}=14^{m} .5\right)$. Part of the mystery can be solved if these carbon stars are related to the Sagittarius dwarf galaxy $\left(\mathrm{SDG} ; \mathrm{m}-\mathrm{M} \simeq 17^{m} \cdot 0\right)$. They are in that case not old and metal-rich, but young, $\sim 0.1 \mathrm{Gyr}$, with SMC-like metallicity ( $\mathrm{Ng} \mathrm{1998).}$

The $\sigma_{R V}=113 \pm 14 \mathrm{~km} \mathrm{~s}^{-1}$ (Tyson \& Rich 1991) radial velocity dispersion of the stars appears to be consistent with Bulge membership. On the other hand, a similar velocity dispersion could be the result from an induced star formation event when the SDG crosses the galactic midplane. It is suggested that the carbon stars are tracers of such an event and that they therefore are located at distances related to the SDG. However, the majority of the carbon stars are not member of the SDG, nor are they similar to the C-stars which are member of the SDG.

The radial velocities can be used to determine a possible membership to the SDG. However, they do not give information about the distance of the stars. In particular, if the stars are located at a distance comparable to the SDG. This implies that only the period-luminosity relation (Groenewegen \& Whitelock 1996) can be used to distinguish unambiguously if the carbon stars are located at Bulge-like or SDG-like distances. Thus far only carbon stars with reliable periods have been identified at a SDG related distance ( $\mathrm{Ng} \&$ Schultheis 1997; Whitelock 1998).
\end{abstract}

\section{Introducing the mystery}

After a long search 34 carbon stars were found by Azzopardi et al. (1991 and references cited therein - ALRW91) in the direction of the Galactic Centre. If located in the metal-rich Bulge they are about $2^{m} \cdot 5$ fainter than the carbon stars from the Magellanic Clouds or the dwarf spheroidal galaxies (Lequeux 1990; Tyson \& Rich 1991 - TR91; Westerlund et al. 1991).

The Bulge is older than the maximum age of $\sim 4 \mathrm{Gyr}$ for a carbon star to be regarded as a genuine TP-AGB star (see $\mathrm{Ng} 1997$ \& 1998 and references cited therein). The ALRW91 C-stars are therefore thought to be located along the RGB as a result of binary evolution. Fig. 1 indicates that for the latter case the stars do not reach the RGB tip. Moreover, the origin of this truncation has not been explained. 


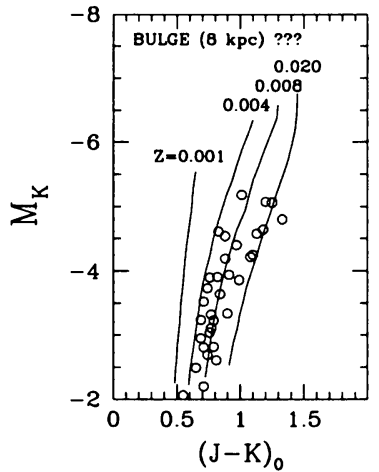

Figure 1. The $\left(\mathrm{M}_{K}, \mathrm{~J}-\mathrm{K}\right)_{0} \mathrm{CMD}$ of the RGB for $10 \mathrm{Gyr}$ isochrones for the metallicities $\mathrm{Z}=0.001,0.004,0.008$, and 0.020 (Bertelli et al. 1994). The absolute magnitudes of the ALRW91 C-stars are determined under the assumption that they are located in the Bulge at a distance of $8 \mathrm{kpc}$. Note that the ALRW91 C-stars do not reach the RGB tip and no explanation has been provided thus far for this behaviour.

The questions raised and answered partly to provide a solution to this standing mystery are: are these stars really located in the Bulge ? Is there an alternative? If so: where are they? and what are the implications?

\section{Relation to the Sagittarius dwarf galaxy ?}

The serendipitous identification of the Sagittarius dwarf galaxy (SDG) was made by Ibata et al. (1994, 1995). Accurate distance determinations from RR Lyrae stars belonging to this galaxy range from $22.0-27.3 \mathrm{kpc}(\mathrm{Ng} 1997$ and references cited therein). The $\sim 2^{m} .5$ difference of the distance modulus between the dwarf galaxy $\left(\mathrm{m}-\mathrm{M}=17^{m} \cdot 0\right)$ and the Galactic Centre at $8 \mathrm{kpc}\left(\mathrm{m}-\mathrm{M}=14^{m} \cdot 5\right)$ lead $\mathrm{Ng} \&$ Schultheis (1997) to suggest that the ALRW91 C-stars could actually be located at the distance of the dwarf galaxy. Note that this does not necessarily imply a SDG membership. Its presence was unknown at the time when the $\mathrm{C}$-stars were identified and a different location could solve the standing question about the origin of the 'Bulge' carbon stars.

\section{Not so metal-rich after all !}

$\mathrm{Ng}(1997 \& 1998)$ analysed this possibility by assuming a relation of the ALRW91 $\mathrm{C}$-stars with the SDG. Once more it is stressed that related $\neq$ membership, except for a small fraction of the C-stars. $\mathrm{Ng}$ demonstrated that the photometric sequence of the ALRW91 C-stars is in that case not exceptional, but comparable with the sequence found for the SMC. In addition, the ALRW91 carbon stars are not metal-rich as previously thought. The metallicity was initially estimated to $\mathrm{Z} \simeq 0.008$ ( $\mathrm{Ng} 1997)$, but a revision has been made to $\mathrm{Z} \simeq 0.004(\mathrm{Ng} 1998)$. $\mathrm{Ng}$ further argued that the stars have an age of $\sim 0.1 \mathrm{Gyr}$.

\section{Elusive radial velocities}

The radial velocities from TR91 for the carbon stars appear to be consistent with a Bulge membership. However, an alternative explanation for the large velocity dispersion is available: induced star formation from the material residing in either the SDG and/or the impact spot in the Galactic disc when the SDG 


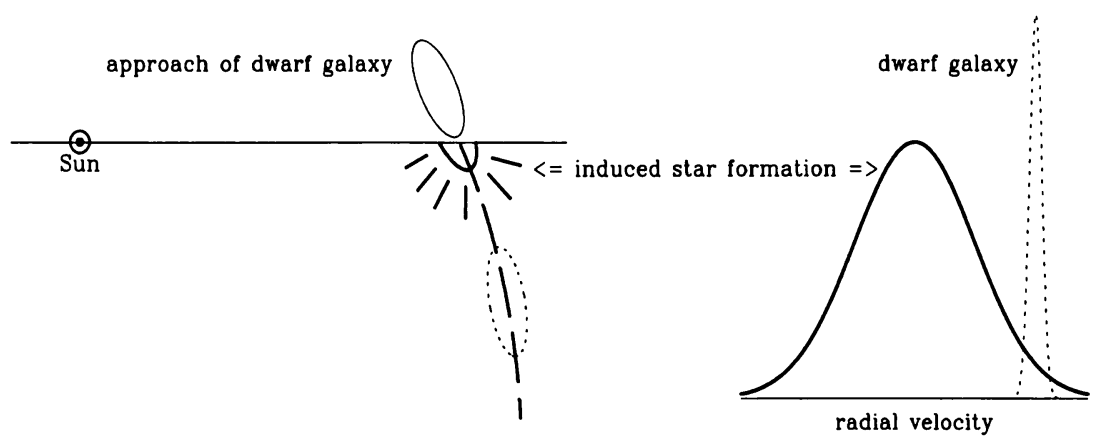

Figure 2. Schematic view of the broad, Bulge like, radial velocity distribution for the material from an induced star formation event, caused by the crossing from a dwarf galaxy through the Galactic midplane. Note that the majority of the new material will not be moving along the dwarf galaxy orbit. The dotted ellipse and the dotted line indicate respectively the current position and radial velocity (not on scale) for the dwarf galaxy

respectively approaches and crosses the galactic plane ( $\mathrm{Ng} \mathrm{1997,1998).} \mathrm{Fig.} 2$ displays a schematic view of the process, where the SDG pushes material out of the Galactic disc ${ }^{1}$. Only a small fraction of the newly formed stars is dragged along the orbit, while another fraction is moving away from the SDG. Note that another fraction of these young stars is moving towards us. This implies that the distribution of radial velocities is considerably broader than expected from a SDG membership alone, i.e. $\sigma_{\mathrm{RV}}>>11.4 \pm 0.7 \mathrm{~km} \mathrm{~s}^{-1}$. The TR91 velocity dispersion of $\sigma_{\mathrm{RV}}=113 \pm 14 \mathrm{~km} \mathrm{~s}^{-1}$ for the ALRW91 C-stars may have been interpreted mistakingly as an indication for a Galactic Bulge membership ${ }^{2}$. Alternatively the large velocity dispersion can also be a signature from an induced star formation event.

\section{Gateway: the galactic disc and the Sagittarius dwarf galaxy}

The impact position at the galactic mid-plane is obtained from the unweighted least-squares fit through the distances determined from the SDG RR Lyrae stars as a function of the galactic latitude $(\mathrm{Ng} \mathrm{1998)}$ and yields $22.8 \mathrm{kpc}$. Adopting

\footnotetext{
${ }^{1}$ Numerical calculations of the structure of the H I disc by Ibata \& Razoumov (1998) indicate that just after impact (their Fig. 2b) material is scattered in all directions above and below the Galactic plane. This should result in a broad radial velocity of the material scattered after the crossing of the Galactic plane. Their Fig. 4 further indicates that part of the gaseous material with $\log T$ in the range $2-4$ should have been converted into stars; some of them possibly comparable to the ALRW91 C-stars.

${ }^{2}$ The uncertainties in the individual velocity values may be as large as $90 \mathrm{~km} \mathrm{~s}^{-1}$. However, if the uncertainties follow a random pattern the mean velocity and its dispersion will not change drastically if the radial velocity values are improved.
} 
a Solar galacto-centric distance of $R_{0}=8.0 \mathrm{kpc}$ implies that the crossing of the SDG occurred at $14.8 \mathrm{kpc}$ distance from the Galactic centre.

To determine the disc metallicity at impact position one has to take the radial dependence of $[\mathrm{Fe} / \mathrm{H}]$ into account. Carraro et al. (1998) determined from open clusters the radial metallicity gradient in different age ranges. The disc metallicity at the crossing position is $[\mathrm{Fe} / \mathrm{H}]=-0.61 \pm 0.20(\mathrm{Z} \simeq 0.0050)$ or $[\mathrm{Fe} / \mathrm{H}]=-0.73 \pm 0.10(\mathrm{Z} \simeq 0.0035)$ with respectively the present day and the average radial metallicity gradient.

Irrespective of the present day or average metallicity gradient it is estimated that the disc metallicity at the crossing position is $\mathrm{Z} \simeq 0.0045 \pm 0.0010$. Within the uncertainties this is in agreement with $\mathrm{Z} \simeq 0.004$.

Considering that an induced star formation event can explain the large velocity dispersion for the ALRW C-stars and taking into account the similarity between the metallicity from a photometric estimate and the disc impact position, it is suggested that the C-stars do not originate from the SDG itself, but are likely formed from material originating from our own Galactic disc.

The average $(\mathrm{J}-\mathrm{K})_{0, S A A O}$ colour of the carbon stars which are members of the dwarf galaxy is $1^{m} .40$ (Whitelock et al. 1996), while the average $(\mathrm{J}-\mathrm{K})_{0, E S O}$ colour of the ALRW91 C-stars is $0^{m} .85$. This immediately shows the marked difference between two groups of carbon stars found in the same direction at a comparable distance. The redder SDG C-stars are likely older and metalricher than the ALRW91 C-stars, because the redder SDG C-stars should be associated with a SDG population comparable or younger than $\sim 4$ Gyr. The youngest population identified for the SDG has an age of about $4 \mathrm{Gyr}$ and a metallicity $\mathrm{Z} \simeq 0.008$ (Mighell et al. 1998).

\section{Why are they still around Sagittarius dwarf galaxy?}

The average radial velocity of $-44 \mathrm{~km} \mathrm{~s}^{-1}$ (TR91) provides a first indication of the distance traveled by the ALRW91 C-stars. Considering a smaller velocity in the past the distance traveled in $0.1 \mathrm{Gyr}$ is $\sim 2.2-3.1 \mathrm{kpc}$. The traveled distance is within the $10 \%-15 \%$ uncertainty of the $22.8 \mathrm{kpc}$ distance to the impact position.

The velocity dispersion of the C-stars provides further an indication about the average separation. Taking into account that the dispersion is smaller, due to a combination of turbulence and a lower velocity in the past, the separation is estimated to $\sim 5.6-8.0 \mathrm{kpc}$. For comparison: the distance between 2 of the 4 globular clusters associated with SDG is about $10 \mathrm{kpc}$, i.e. Terzan 8 at $21.1 \mathrm{kpc}$ and Arp 2 at $31.0 \mathrm{kpc}$ (Da Costa \& Armandroff 1995), while the distance between the two carbon stars with well determined periods is about $5 \mathrm{kpc}$, i.e. $21.9 \mathrm{kpc}$ for a carbon semiregular variable ( $\mathrm{Ng} \&$ Schultheis 1997$)$, and $26.7 \mathrm{kpc}$ for a carbon Mira (Whitelock 1998).

Both the average distance traveled by the ALRW91 C-stars and the separation between them are such that most of the stars can still be associated with the Sagittarius dwarf galaxy. 


\section{Still mysterious ???}

The general accepted models of the SDG orbit indicate (see Figure 11 in Ibata et al. 1997 and references cited therein), together with the proper motion reported by Irwin et al. (1996), that the SDG is still moving towards the galactic mid-plane. On the other hand, the ALRW91 C-stars appear to form the factual evidence that the SDG has already crossed the galactic mid-plane. In addition, the orbit at low galactic latitudes (Figure 4 in $\mathrm{Ng} \mathrm{1997)} \mathrm{is} \mathrm{not} \mathrm{consistent} \mathrm{with}$ the position obtained from RR Lyrae stars, which might partly be due to uncertainties in the extinction. A study of the Galactic globular cluster Palomar 5 (Scholz et al. 1998) further indicates that the cluster is moving in the opposite direction with respect to the Ibata et al. orbital motion of the SDG.

Instead of looking for alternative explanations for the apparent contradiction, one might consider a re-analysis of the orbit combined with an independent verification of the proper motion. A revised orbit and proper motion might show that the globular cluster Palomar 5 is tidally stripped from the SDG (Lin 1996) and also associate the Smith high velocity Cloud with the SDG (Bland-Hawthorn et al. 1998).

The models thus far consider only the gravitational interaction between the Galaxy and the SDG (Ibata \& Lewis 1998; Nair \& Miralda-Escudé 1998), but do not take into account an encounter, induced, star formation event. Improved models should therefore be employed to trace the stars from such an event. In addition, a detailed proper motion study and a renewed determination of the radial velocities of the ALRW91 C-stars are still required to explain and to understand better the presence of the carbon stars.

\section{Conclusion}

The 'Bulge' (ALRW91) carbon stars are located at a distance comparable to the Sagittarius dwarf galaxy. However, the majority of these stars $(\mathrm{Z} \simeq 0.004$, $\mathrm{t} \simeq 0.1 \mathrm{Gyr}$ ) are not member of the Sagittarius dwarf galaxy nor are they comparable with the carbon stars found in the Sagittarius dwarf galaxy $(\mathrm{Z} \simeq 0.008$, $\mathrm{t} \simeq 4 \mathrm{Gyr}$; see for details $\mathrm{Ng} 1998$ ).

The radial velocities cannot be used reliably as an argument in favour of Bulge membership, due to a possible confusion with the velocity dispersion of the stars resulting from a crossing induced star formation event which scatters new stars in all directions ${ }^{3}$.

If there are carbon stars in the Bulge then the carbon star period-luminosity relation (Groenewegen \& Whitelock 1996) should be employed to determine their distances. Up to date no carbon semiregular or Mira variable has been reported with a period-luminosity which unambiguously associates it with the Galactic Bulge.

\footnotetext{
${ }^{3}$ Bonus: the microlensing events between the stars from the Bulge/bar and the induced semisphere of young stars, due to the crossing of the SDG through the galactic midplane, could well prove to contribute significantly to the surprisingly high event rate found towards the Galactic. Centre (Paczyński 1996 and references cited therein).
} 
Acknowledgments. $\mathrm{Ng}$ acknowledges a travel grant received from the IAU. The research is supported by grant ERBFMRX-CT96-0086 from the TMR network 'Formation and evolution of galaxies' of the European Community.

\section{References}

Azzopardi M., Lequeux J., Rebeirot E., Westerlund B.E., 1991, A\&AS 88, 265 (ALRW91)

Bertelli G., Bressan A., Chiosi C., Fagotto F., Nasi E., 1994, A\&AS 106, 275

Bland-Hawthorn J., Veilleux S., Cecil G.N., et al., 1998, MNRAS 299, 611

Carraro G., Ng Y.K., Portinari L., 1998, MNRAS 296, 1045

Da Costa G.S., Armandroff T.E., 1995, AJ 109, 2533

Groenewegen M.A.T., Whitelock P.A., 1996, MNRAS 281, 1347

Ibata R.A., Lewis G.F., 1998, ApJ 500, 575

Ibata R.A., Razoumov A.O., 1998, A\&A 336, 130

Ibata R.A., Gilmore G., Irwin M.J., 1994, Nature 370, 194

Ibata R.A., Gilmore G., Irwin M.J., 1995, MNRAS 277, 781

Ibata R.A., Wyse R.F.G., Gilmore G., et al., 1997, AJ 113, 635

Irwin M., Ibata R., Gilmore G., et al., 1996, in 'Formation of the galactic halo ... inside and out', ASP Conf. Series 92, H. Morrison and A. Sarajedini (eds.), p. 84

Lequeux J., 1990, in 'From Miras to planetary nebulae: which path for stellar evolution?', M.-O. Mennessier and A. Omont (eds.), Editions Frontières, p. 273

Lin D.N.C., 1996, in 'Gravitational dynamics', O. Lahav, E. Terlevich and R.J. Terlevich (eds.), Cambridge University Press, p. 15

Mighell K., Armandroff T., Sarajedini A., et al., 1998, in 'Galaxy interaction at low and high redshift', IAU Symposium 186, ed. D.B. Sanders, Kluwer, in press

Nair V., Miralda-Escudé J., 1998, ApJ submitted (astro-ph/9805278)

Ng Y.K., 1997, A\&A 328, 211

Ng Y.K., 1998, A\&A 338, 435

Ng Y.K., Schultheis M., 1997, A\&AS 123, 115

Paczyński B., 1996, ARA\&A 34, 419

Scholz R.-D., Irwin M., Odenkirchen M., Meusinger H., 1998, A\&A 333, 531

Tyson N., Rich R.M., 1991, ApJ 367, 547 (TR91)

Westerlund B.E., Lequeux J., Azzopardi M., Rebeirot E., 1991, A\&A 244, 367

Whitelock P.A., 1998, in 'Pulsating Stars - Recent Developments in Theory and Observation', M. Takeuti and D. Sasselov (eds.), Universal Academic Press, Tokyo, in press

Whitelock P.A., Irwin M., Catchpole R.M., 1996, New Astronomy 1, 57 